\title{
Tamiz metabólico neonatal por espectrometría de masas en tándem: dos años de experiencia en Nuevo León, México
}

\author{
María del Rosario Torres-Sepúlveda, QCB, (I) Laura E Martínez-de Villarreal, MC,(I) \\ Carmen Esmer, MC,(I) Rogerio González-Alanís,(I) Consuelo Ruiz-Herrera, QCB,(I) Alejandra Sánchez-Peña, Lic en Nutr, (I) \\ José Alberto Mendoza-Cruz, MC, ${ }^{(2)}$ Jesús ZVillarreal-Pérez, MC.(3)
}

\author{
Torres-Sepúlveda MR, Martínez-de Villarreal LE, \\ Esmer C, González-Alanís R, Ruiz-Herrera C, \\ Sánchez-Peña A, Mendoza-Cruz JA, Villarreal-Pérez JZ. \\ Tamiz metabólico neonatal por espectrometría \\ de masas en tándem: dos años \\ de experiencia en Nuevo León, México. \\ Salud Publica Mex 2008;50:200-206.
}

\begin{abstract}
Resumen
Objetivo. Instituir un programa estatal de tamizaje neonatal ampliado para identificar errores innatos del metabolismo y determinar su prevalencia en la población de recién nacidos del estado de Nuevo León. Material y métodos. Entre marzo de 2002 y febrero de 2004 se incluyeron neonatos consecutivos nacidos en hospitales públicos del estado. Se colectaron muestras de sangre en papel filtro entre las 24 y 48 horas de vida y se las sometió a tamiz metabólico mediante espectrometría de masas en tándem. Resultados. Se analizaron 42264 primeras muestras y se detectaron siete casos, uno de cada padecimiento: homocistinuria, fenilcetonuria, citrulinemia, tirosinemia/transitoria, deficiencia de 3-metilcrotonil-CoA carboxilasa, deficiencia de 3-hidroxi-3metilglutaril-CoA liasa y galactosemia típica. Conclusiones. La incidencia acumulada de defectos metabólicos en la población fue de 1:5 000 con $0.22 \%$ de casos falso-positivos. El programa permitió identificar y tratar con oportunidad los trastornos metabólicos al nacimiento con una efectiva prevención secundaria del retraso mental.
\end{abstract}

Palabras clave: tamizaje neonatal; errores innatos del metabolismo; prevención de discapacidad; retraso mental; retraso del desarrollo; México
Torres-Sepúlveda MR, Martínez-de Villarreal LE, Esmer C, González-Alanís R, Ruiz-Herrera C, Sánchez-Peña A, Mendoza-Cruz JA,Villarreal-Pérez JZ. Expanded newborn screening using tandem mass spectrometry: Two years' experience in Nuevo León, Mexico.

Salud Publica Mex 2008;50:200-206.

\begin{abstract}
Objective. To initiate a statewide expanded metabolic screening program in neonates with the purpose of identifying the most common inborn errors of metabolism. Material and Methods. From March 2002 through February 2004, a blood sample was obtained between 24 and 48 hours after delivery from every consecutive child born in public hospitals in Nuevo León. It was spotted on filter paper and analyzed by tandem mass spectrometry for expanded metabolic screening. Results.A total of 42264 samples were analyzed.Were obtained seven positive results, one for each disorder:homocystinuria, hyperphenylalaninemia, citrulinemia, transient tyrosinemia, 3-methylcrotonyl CoA carboxylase deficiency, 3-hydroxy-3-methylglutaryl CoA deficiency, and classic galactosemia. Conclusions. The estimated incidence of inborn errors of metabolism is 1:5 000, with a false positive rate of $0.22 \%$. The program permitted the identification of metabolic disorders in the newborn, allowing an early intervention and prevention of life-threatening events and permanent neurological damage.
\end{abstract}

Key words: metabolic neonatal screening; inborn errors of metabolism; prevention; mental retardation; developmental disabilities; Mexico

(I) Departamento de Genética, Facultad de Medicina, Universidad Autónoma de Nuevo León, México.

(2) Servicios de Salud de Nuevo León, México.

(3) Servicio de Endocrinología, Hospital Universitario Dr. José E. González. Nuevo León, México.

Fecha de recibido: 9 de agosto de 2007 - Fecha de aceptado: 4 de diciembre de 2007

Solicitud de sobretiros: Dra. Laura E. Martínez de Villarreal. Departamento de Genética, Facultad de Medicina, Universidad Autónoma de Nuevo León. Av. Madero y Eduardo Aguirre Pequeño s/n Col. Mitras Centro. 64460 Monterrey, Nuevo León, México.

Correo electrónico: laurmart@fm.uanl.mx, laelmar@yahoo.com.mx 
$E^{1}$ tamiz metabólico neonatal (TMN) para la detección de errores innatos del metabolismo (EIM) es una medida de salud pública dirigida a identificar los trastornos con graves consecuencias en el recién nacido (RN). Dada la importancia del diagnóstico oportuno de los EIM, para reducir la morbi-mortalidad y prevenir la presencia de retraso mental, la mayoría de los países desarrollados lleva a cabo programas de TMN. El tamiz en el recién nacido se inició en 1960 en Estados Unidos, con la utilización de gotas de sangre seca en papel filtro para la detección de fenilcetonuria mediante la prueba de inhibición bacteriana de Guthrie; a partir de entonces ha sido posible reconocer algunos trastornos del metabolismo de aminoácidos como la enfermedad de orina con olor a jarabe de arce, la hipermetioninemia y la tirosinemia. ${ }^{1,2}$

El número y tipo de trastornos que se detectan al nacer varían entre cada país e incluso dentro de una misma nación de acuerdo con la reglamentación o normatividad existentes, recursos económicos y la frecuencia de las enfermedades en cada población. Cuando se pretende introducir un programa de tamizaje neonatal, se deben seguir los criterios de Wilson y Jungner que publicó la Organización Mundial de la Salud y en los cuales se establece que la enfermedad debe tener una incidencia elevada, posibilidad de un tratamiento médico efectivo, existencia de una prueba de tamizaje barata con alta sensibilidad y especificidad para un gran volumen de muestras y una relación costo-beneficio razonable para identificar y tratar de forma oportuna estos trastornos. ${ }^{3}$

Cuando no se dispone de la información sobre la incidencia de los padecimientos en la población, es importante iniciar un programa piloto con la finalidad de incluir una muestra poblacional grande y analizar el mayor número de enfermedades posible. De manera inicial, la metodología empleada para el tamizaje de estas enfermedades era económica pero muy lenta y ello llevó al desarrollo de técnicas más sensibles y rápidas, como el radioinmunoensayo, inmunoensayo enzimático, enfoque isoeléctrico, cromatografía de líquidos de alta resolución, fluorometría y ensayos enzimáticos directos. Sin embargo, estas pruebas se enfocan en el reconocimiento de un solo trastorno metabólico, de tal manera que su empleo para los programas de tamiz neonatal ampliado no resulta eficiente en términos del costo. ${ }^{2,4}$ La espectrometría de masas en tándem (MS/ MS) permite el análisis de múltiples compuestos para la identificación de diversas enfermedades en una pequeña cantidad de sangre del talón del recién nacido, razón por la cual su obtención carece de riesgos y quizá ofrezca una mayor eficiencia y menor costo de la prueba. ${ }^{5}$ Una de las múltiples ventajas de esta metodología es que no se requieren separaciones cromatográficas previas, que consumen tiempo debido a que la separación y el reconocimiento de concentraciones en el análisis ocurren de modo simultáneo en el mismo instrumento; además, el límite de detección es hasta de $1 \mathrm{nmol} / \mathrm{ml}$ de sangre. ${ }^{6}$ Algunas modificaciones en la técnica hacen posible la detección de trastornos del metabolismo de ácidos grasos, ácidos orgánicos y aminoácidos con alta sensibilidad y especificidad. ${ }^{78}$ La técnica tiene tantas aplicaciones que permite el análisis de otros compuestos químicos, para lo cual sólo basta cambiar los parámetros de barrido de tal manera que es posible identificar más de 30 trastornos metabólicos. ${ }^{9}$

En este trabajo se presentan los resultados de los primeros 24 meses de un programa de cobertura estatal que inició en el año 2002 por una iniciativa de la Secretaría de Salud en el estado de Nuevo León, México, para realizar TMN ampliado de casi 30 trastornos del metabolismo. El objetivo fue instituir un programa estatal de tamizaje neonatal ampliado para identificar errores innatos del metabolismo y determinar su prevalencia en la población de recién nacidos del estado mencionado.

\section{Material y métodos}

$\mathrm{Al}$ inicio del estudio se solicitó a las instituciones de salud participantes que las madres y sus hijos permanecieran en el hospital o clínica al menos por 24 horas; se capacitó al personal de enfermería en la forma de obtener la muestra, se coordinó el envío y recepción en el laboratorio, se estandarizó el análisis por MS / MS y se obtuvieron los resultados en una semana. También se estableció una red entre el primer y segundo niveles de atención para localizar a la brevedad a los recién nacidos con resultados positivos para la toma de una segunda muestra.

\section{Población de estudio}

El comité de ética institucional aprobó el estudio. Se incluyó a todos los recién nacidos consecutivos, previa aceptación y firma de consentimiento informado por los padres o tutores; los niños nacieron en los hospitales pertenecientes a la Secretaría Estatal de Salud (Hospitales Generales de Sabinas, Galeana, Cerralvo, Montemorelos y Dr. Arroyo, Hospital Metropolitano) y Hospital Universitario. Las muestras de sangre se obtuvieron por punción del talón del recién nacido a las 24 horas de vida en la sala de cunas, mientras que a los niños que ingresaron en la unidad de cuidados intensivos pediátricos se les tomó una muestra a las 24 horas y otra al mes de vida o al darlos de alta. La 
sangre se colocó en papel filtro $S \& S$ 903, se dejó secar a temperatura ambiente y se envió al laboratorio de genética bioquímica en el Departamento de Genética de la Facultad de Medicina de la Universidad Autónoma de Nuevo León. Se obtuvieron datos demográficos de todos los recién nacidos: nombre de la madre, género, lugar de origen, dirección, fecha de nacimiento, edad gestacional, peso, tipo de alimentación, fecha y hora de la toma y folio o número de registro.

\section{Preparación y análisis de la muestra}

De cada muestra de sangre se obtuvieron círculos de 3 $\mathrm{mm}$ de diámetro ( $3 \mu \mathrm{l}$ de sangre), se colocaron dentro de los pozos de una placa de poliestireno de 96 celdillas y se añadieron $100 \mu \mathrm{l}$ de una solución metanólica con 12 estándares internos de isótopos estables para acilcarnitinas y 12 para aminoácidos (Neogram aminoacids and acylcarnitines tandem mass spectrometry kit). Las placas se incubaron y agitaron a $30^{\circ} \mathrm{C}, 750 \mathrm{rpm}$ por 30 minutos. Se tomaron $65 \mu$ ldel extracto metabólico, se transfirieron a una placa de polipropileno y se secaron bajo una corriente de nitrógeno (MicroDS96 Porvair). Los derivados butilésteres se prepararon por incubación con $50 \mu \mathrm{l}$ de butanol/HCL 3N, por 30 minutos a $65^{\circ} \mathrm{C}$. Después de eliminar el reactivo derivatizante con nitrógeno, los extractos derivatizados se reconstituyeron con $75 \mu \mathrm{l}$ de solución de reconstitución, se cubrieron con papel aluminio y se analizaron en el MS/MS. Los aminoácidos y acilcarnitinas se analizaron en un espectrómetro de masas de triple cudrupolo API 2000 (Perkin Elmer Sciex), acoplado a una microbomba y un automuestreador Series 200 (Perkin Elmer). Se inyectaron $3 \mu \mathrm{l}$ de cada muestra dentro de la cámara de ionización a una velocidad de flujo de $50 \mu \mathrm{l}$ por minuto. Se empleó el método de adquisición de datos, que incluye "barrido total" (full scan) y reacción de monitoreo múltiple (MRM).

Los datos se obtuvieron y procesaron con el software Analyst y la base de datos Neogram. Después del análisis de MS / MS se efectuó la conversión del espectro de masas a resultados clínicamente significativos mediante el cálculo de la concentración de cada analito. La relación de la intensidad de un metabolito desconocido con su respectivo estándar interno produce una relación de iones. La relación se convierte a concentración con el software Neogram. Los resultados se expresaron en micromoles por litro $(\mu \mathrm{M} / \mathrm{L})$. Se consideraron como indicadores de enfermedad sólo los metabolitos ya informados en las publicaciones que orientan hacia un trastorno metabólico específico (cuadro I) ${ }^{10}$ Se calcularon los índices de varios analitos: fenilalanina/ tirosina, leucina/fenilalanina, metionina/fenilalanina, propionil/acetilcarnitina, octadecanoilcarnitina/deca- noilcarnitina por ser de utilidad para el diagnóstico de algunos trastornos. ${ }^{11,12}$

Los niveles de galactosa se cuantificaron con el fluorómetro (VictorD de Wallac) y el estuche de galactosa total (Total Galactose Kit, Perkin Elmer).

\section{Valores de corte}

A partir de los resultados de las primeras 500 muestras se obtuvieron la media y desviación estándar para cada uno de los compuestos analizados y se establecieron los valores de referencia para la población. Los resultados de 3 a 4 desviaciones estándar se consideraron como "alerta" y los mayores de 4 como "urgentes". Se realizó un reajuste de los valores de corte a las 10000 muestras; algunos de estos valores se incluyen en el cuadro II. Por ejemplo, para la galactosa, los valores de 7 a $10 \mathrm{mg} / \mathrm{dl}$ se consideraron "alerta" y los mayores de $10 \mathrm{mg} / \mathrm{dl}$ como "urgentes". El estudio se llevó a cabo mediante controles de calidad internos del laboratorio, el cual está adscrito a los Programas de Control y Aseguramiento de la Calidad para tamiz neonatal del Centro de Control de Enfermedades de Atlanta (CDC), que evalúa cada cuatro y seis meses a los laboratorios participantes.

\section{Seguimiento de resultados anormales}

Ante un resultado anormal se repitió el análisis en la misma muestra, en los casos en los que se repetía un resultado anormal, y se consideró de relevancia clínica; asimismo, se solicitó una segunda muestra y la revisión inmediata por el pediatra. Si el resultado anormal se repetía en la segunda muestra, se realizaban pruebas diagnósticas confirmatorias.

\section{Resultados}

Entre marzo del año 2002 y febrero del 2004 se analizaron 42264 muestras y se registraron 1388 resultados anormales. Se analizaron 1231 segundas muestras; de ellas, $144(12 \%)$ repitieron un resultado anormal. Sólo fue posible concluir sobre su estado de normal o anormal en 102 pacientes (figura 1), de los cuales se confirmaron siete trastornos metabólicos y los 95 restantes $(0.22 \%)$ se consideraron falso-positivos dado que no presentaron anormalidad clínica o bioquímica persistente; 42 casos permanecieron en seguimiento sin una conclusión definitiva en cuanto a su diagnóstico metabólico. Los diagnósticos que se confirmaron fueron citrulinemia, tirosinemia transitoria, homocistinuria, hiperfenilalaninemia, deficiencia aislada de 3-metilcrotonil-CoA carboxilasa (3MCC), deficiencia de 3-hidroxi-3-metilglutaril= CoA liasa (HMG) y galactosemia común, un 


\section{Cuadro I}

Frecuencia de ERRores INNATOS del METABolismo EN RECIÉn NACIDOS CON MARCADORES PRIMARIOS Y SECUNDARIOS*

Trastorno

Marcador primario

Marcador secundario

Frecuencia en recién nacidos $\S$

Aminoacidemias

\begin{tabular}{|c|c|c|c|}
\hline Fenilcetonuria & Fenilalanina & Tirosina & $\mathrm{I}: 25000$ \\
\hline Enfermedad de orina con olor de arce & Leucina & Isoleucina, valina & $\mathrm{I}: 250000$ \\
\hline Homocistinuria & Metionina & & $\mathrm{I}: 250000$ \\
\hline Tirosinemia II & Tirosina & & $\mathrm{I}: 250000$ \\
\hline Citrulinemia & Citrulina & & $\mathrm{I}: 125000$ \\
\hline Hiperornitinemia & Ornitina & & 100 casos \\
\hline Argininemia & Arginina & & $\mathrm{I}: 300000$ \\
\hline Aciduria argininosuccínica & Citrulina & Ornitina & $\mathrm{I}: 250000$ \\
\hline
\end{tabular}

Trastornos de la oxidación de ácidos grasos

Deficiencia de acil-CoA deshidrogenasa de cadena media (MCAD)

Aciduria glutárica tipo II (MADD)

$\begin{array}{lll}\mathrm{C} 8 & \mathrm{C} 6, \mathrm{Cl}, \mathrm{Cl} 0: \mathrm{I} & \mathrm{I}: 250000 \\ \mathrm{C} 8 & \mathrm{C} 4, \mathrm{C} 5, \mathrm{Cl}, \mathrm{Cl} 2, \mathrm{Cl} 4, \mathrm{Cl} 6 & \mathrm{I}: 250000 \\ \mathrm{Cl} 4: \mathrm{C} & \mathrm{Cl} 4, \mathrm{Cl} 6 & \mathrm{I}: 250000 \\ \mathrm{C} 4 & & 1: 250000 \\ \mathrm{Cl} 6 \mathrm{OH} & \mathrm{Cl} 8: \mathrm{IOH}, \mathrm{Cl} 8 \mathrm{OH}, \mathrm{Cl}, \mathrm{Cl} 4, \mathrm{Cl} 4: \mathrm{I} & \mathrm{I}: 250000 \\ \mathrm{Cl} 6 & \mathrm{C} 0, \mathrm{Cl}, \mathrm{Cl} 8: \mathrm{I} & \mathrm{I}: 250000\end{array}$

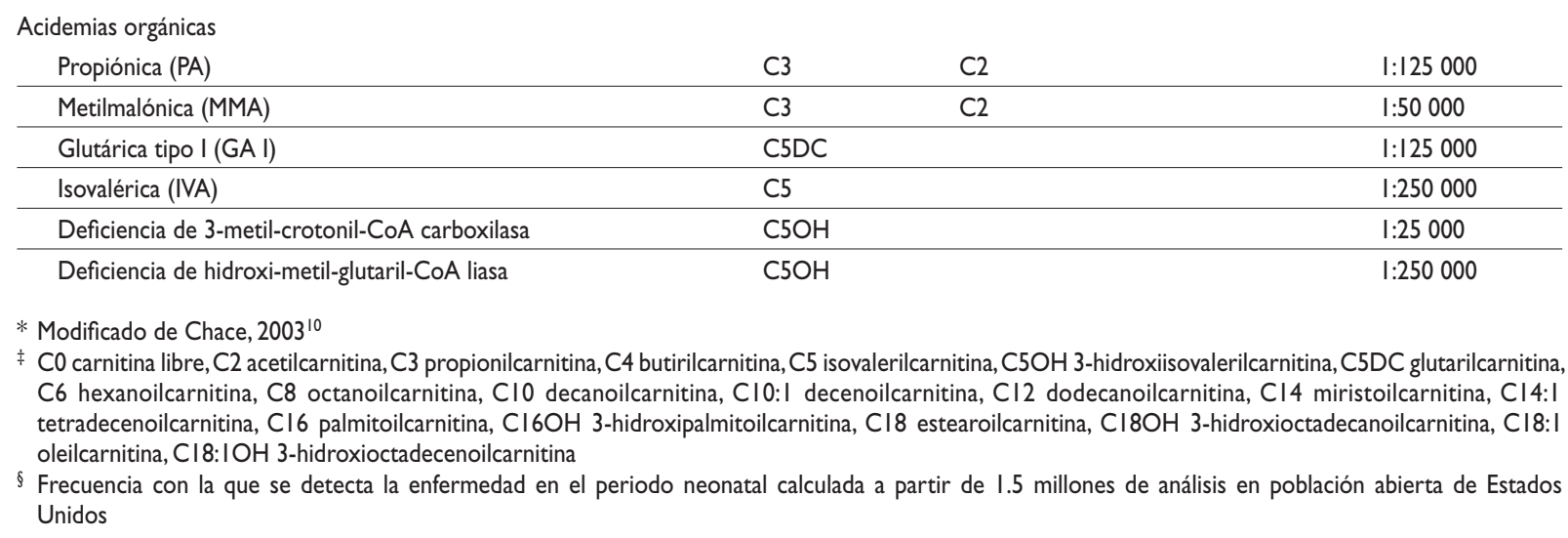

caso por cada diagnóstico; tales casos se sometieron a mediciones periódicas por el laboratorio y control multidisciplinario por pediatras, nutriólogos y genetistas. Estos pacientes recibieron terapia nutricional y medicamentos cuando fue requerido. Hasta la fecha, con excepción del recién nacido con deficiencia de 3-hidroxi3-metilglutaril=CoA liasa (HMG) que falleció, el resto permanece asintomático, sin secuelas neurológicas.

Se identificó a cinco neonatos con elevación de 3hidroxi-isovaleril-carnitina $(\mathrm{C} 5 \mathrm{OH})$. En uno de ellos se confirmó el diagnóstico de deficiencia de 3-MCC, otro se trató de un caso de deficiencia de HMG confirmado con determinación de ácidos orgánicos y de los tres casos restantes, la elevación fue secundaria a una deficiencia de 3-MCC materna asintomática y los otros dos se consideraron falso-positivos debido a que la segunda muestra fue normal. Además, se encontró $0.16 \%$ (56 casos) con ácido aspártico elevado, sin que esta elevación tuviera significado clínico.

\section{Discusión}

En 1986 se inició en México la detección de hipotiroidismo congénito y fenilcetonuria en hospitales que atienden a RN; a partir de entonces en diferentes estados (sobre todo en el Distrito Federal) se iniciaron programas de 
Cuadro II

VALORES DE CORTE PARA ALGUNOS AMINOÁCIDOS Y ACILCARNITINAS OBTENIDAS DE 10000 MUESTRAS en el presente estudio.* Nuevo León, México

\begin{tabular}{lcll} 
Aminoácidos & $\begin{array}{c}\text { Valor de corte } \\
\mu \text { mol/L }\end{array}$ & \multicolumn{1}{c}{ Acilcarnitinas } & $\begin{array}{c}\text { Valor de corte } \\
\mu \text { mol/L }\end{array}$ \\
Alanina & 532.50 & Carnitina & 93.45 \\
\hline Citrulina & 44 & Acetilcarnitina & 35 \\
\hline Glicina & 1020 & Propionilcarnitina & 8 \\
\hline Leucina/isoleucina & 450 & Butirilcarnitina & 1.4 \\
\hline Metionina & 132.75 & Isovalerilcarnitina & 1.19 \\
\hline Fenilalanina & 120.5 & 3-hidroxi-isovaleril carnitina & 0.8 \\
\hline Tirosina & 309.25 & Octanoilcarnitina & 0.4 \\
* Laboratorio de Genética Bioquímica, Departamento de Genética, Facultad \\
de Medicina, Universidad Autónoma de Nuevo León, 2002
\end{tabular}

TMN que incluían la búsqueda sola de hipotiroidismo; otros agregaban fenilcetonuria y algunos más diversas enfermedades. ${ }^{13,14}$ Con posterioridad, en octubre de 2002, se emitió la Norma Oficial Mexicana para la prevención y control de los defectos al nacimiento, en la cual se establece que todos los hospitales que atienden partos y recién nacidos deberán realizar la toma de muestra para TMN (NOM-034.SSA2-2002). ${ }^{15}$

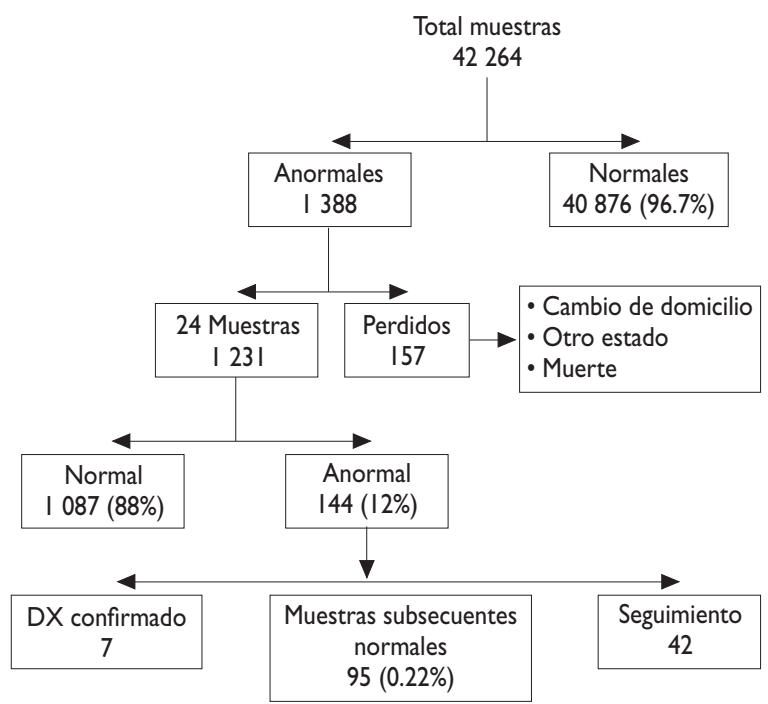

Figura I.Resultados del Programa de Tamiz Neonatal POR ESPECTROMETRÍA DE MASAS EN TÁNDEM EN EL PRESENTE estudio. 2002-2004, Nuevo León, MéXico
Desde julio de 1999, la Secretaría de Salud del estado de Nuevo León, junto con el Departamento de Genética de la Facultad de Medicina, inició el TMN en orina; éste, además de una baja cobertura, muestra un alto porcentaje de falso-positivos por diversas razones (interferencia con medicamentos, dificultad en la toma de muestra, procesamiento prolongado de la muestra, entre otros). Por esta razón, en 2002 se decidió iniciar un programa de TMN en sangre mediante MS/MS. La introducción de MS/MS no sólo mejora la sensibilidad y especificidad del tamiz neonatal, sino que permite un perfil amplio y automatizado que detecta múltiples trastornos de manera simultánea y en un tiempo corto (2.5 minutos por muestra). Siempre que se aplican nuevas tecnologías, los laboratorios deben establecer sus propias condiciones para analizar las muestras y los valores de referencia para la población en estudio, de tal modo que cumplan con los requisitos preestablecidos de aseguramiento y control de la calidad; algunos de los valores que se obtuvieron en el presente estudio se muestran en el cuadro II que difieren respecto de otros países. ${ }^{16} \mathrm{El}$ tamiz metabólico en niños mayores tiene el inconveniente de que las concentraciones de los analitos muestran variación posnatal, por lo que cada laboratorio debe contar con valores de corte apropiados para cada edad. ${ }^{17,18}$

Este trabajo presenta los resultados de un programa de tamiz neonatal para más de 30 padecimientos metabólicos e incluye el mayor número de recién nacidos sanos estudiados hasta la fecha, informados en la bibliografía nacional. En este protocolo se incluyeron a 42264 $\mathrm{RN}$, lo que representa $23 \%$ del total de nacimientos que en el estado de Nuevo León alcanzan los 90 000/año; alrededor de $20 \%$ se atiende en hospitales públicos de la Secretaría de Salud del estado, por lo que se consiguió una cobertura casi total. En este estudio se halló una frecuencia calculada de EIM de 1:5000, lo que se asemeja a lo encontrado en otros países, como Estados Unidos ${ }^{16}$ y Australia (1:4 000-4 500). ${ }^{19}$ Sin embargo, existen naciones como Arabia Saudita (1:1381) con una prevalencia alta de estos padecimientos. ${ }^{11,20}$

La prevalencia de los EIM en cada población puede variar por características genéticas de la población, grado de consanguineidad, efecto de fundador, etc. Otros factores que modifican la prevalencia global son la cantidad y tipo de enfermedades que se incluyen en el tamiz; por ejemplo, algunos detectan fibrosis quística, deficiencia de biotinidasa o deshidrogenasa de glucosa6-fosfato, que son enfermedades de alta incidencia y representan una mayor prevalencia global de EIM. ${ }^{19,20}$ El perfil de enfermedades identificado en este estudio incluyó algunas enfermedades bastante raras con una frecuencia en otras poblaciones de 1:60 000 a 1:100 000, 
por lo que se considera que aumentar el tamaño de la muestra permite reconocer trastornos menos frecuentes y determinar con base en su frecuencia los trastornos que deben incluirse en el tamiz. Las anormalidades detectadas en los casos tamizados, como la hiperfenilalaninemia, la elevación de la tirosina y la hiperhomocistinemia, coinciden con los errores innatos del metabolismo de mayor prevalencia mundial. Con la metodología MS / MS también se identificó un RN con citrulinemia, un padecimiento raro (cuadro I) que ocasiona problemas neurológicos graves e hiperamonemia; en este caso, con el tratamiento oportuno y el seguimiento nutricio, permanece libre de manifestaciones de la enfermedad.

Otros dos casos, uno con deficiencia de 3-MCC y otro con deficiencia de HMG, se reconocieron en el TMN por elevación de la concentración de $\mathrm{C} 5 \mathrm{OH}$. Esta anormalidad en algunos recién nacidos se vincula con diferentes EIM, por ejemplo las deficiencias de 3-metil-crotonil-CoA carboxilasa (3MCC), 3-hidroxi-3-metilglutaril-CoA liasa (HMG), betacetotiolasa, 2-metil-3-hidroxibutiril-CoA deshidrogenasa, hidratasa-3-metilglutacónica, biotinidasa y holocarboxilasa sintasa. En un estudio realizado por Berry y colaboradores (comunicación personal) también se encontró este tipo de casos con elevación de $\mathrm{C} 5 \mathrm{OH}$ $(0.019 \%)$, entre los que se confirmó deficiencia de 3MCC, deficiencia parcial de biotinidasa y deficiencia HMG. El fenotipo de esas anomalías se extiende desde individuos asintomáticos hasta episodios agudos de acidosis metabólica que ponen en peligro la vida, con deterioro subsecuente y muerte. Otro hallazgo fue la presencia de ácido aspártico elevado en $0.16 \%$ de las muestras, pero esta anormalidad no se considera patológica.

La detección neonatal de galactosemia se incluye en la mayor parte de los TMN ampliados; este protocolo encontró un caso entre los 42 264; la intervención temprana con suspensión de galactosa de la dieta permitió que el paciente muestre aun hoy buena evolución.

El análisis sistematizado de los resultados y la retroalimentación de los estudios confirmatorios permitieron conocer la existencia de resultados falso-positivos $(0.22 \%)$, que pueden atribuirse a las condiciones de colección, traslado o almacenamiento de la muestra, o bien, a datos inexactos del caso sobre su edad gestacional, tipo de alimentación (parenteral o dietas especiales), ingestión de fármacos (ácido piválico, anticonvulsivos), transfusiones, etc. ${ }^{10,20}$ Un resultado falso-positivo genera angustia en los padres por la espera para un estudio confirmatorio, por lo que es importante que el médico les informe que un resultado positivo en el tamiz no significa en todos los casos que el niño sufra la enfermedad.
En algunas afecciones como las aminoacidopatías es necesario que el recién nacido experimente antes una exposición a la alimentación, por lo que el tiempo ideal para la toma de la muestra fluctúa entre 24 y 72 horas, lo cual no siempre se logra debido a que los sistemas hospitalarios requieren dar de alta a los recién nacidos antes de las 24 horas y en ocasiones los niños regresan a las salas de urgencias ya con síntomas de la enfermedad metabólica. ${ }^{19}$ En algunos trastornos metabólicos las manifestaciones son tardías y no es posible detectarlas mediante TMN, de tal manera que las características de dicha población dificultan contar con sistemas de seguimiento de casos que permitan establecer la prevalencia de los resultados falso-negativos.

El TMN no sólo es una prueba más de laboratorio, sino una medida con varios componentes que incluyen el número de enfermedades diagnosticadas, el seguimiento personalizado de los casos, la necesidad de contar con pruebas diagnósticas confirmatorias, educación del personal médico y la población en general, ${ }^{20}$ atención interdisciplinaria para la intervención oportuna y adecuada y, sobre todo, contar con un laboratorio con la experiencia y certificaciones necesarias para ofrecer resultados confiables. En el corto plazo se analizará la necesidad de incluir dentro del tamizaje neonatal otras enfermedades como biotinidasa, 17-hidroxiprogesterona para hiperplasia suprarrenal y tripsinógeno inmunorreactivo para fibrosis quística, que ya se han incorporado en otros países.

La aplicación exitosa del MS/MS al programa del TMN debe incluir un control estricto del método de preparación de la muestra, configuración y mantenimiento apropiado del instrumento, un perfil de interpretación adecuado de los resultados, cumplimiento y seguimiento de los sistemas de control de calidad y, en particular, la experiencia del personal del laboratorio. ${ }^{21,22}$

Este programa permitió la detección temprana e intervención oportuna en los casos positivos y evitar la morbilidad adjunta de estos trastornos, que de otra manera conducirían a la discapacidad.

\section{Agradecimientos}

Los autores expresan su agradecimiento a las siguientes personas por su participación en este estudio: Dr. Antonio Velázquez y Dra. Marcela Vela Amieva, MC. Isabel Ibarra González, del Instituto Nacional de Pediatría; la Dra. Enid Rangel Córdova, del Departamento de Genética y la Dra. Alma Rosa Marroquín del Hospital Universitario Dr. José E. González; además de todo el personal de los Servicios de Salud de Nuevo León. 


\section{Referencias}

I. MacCready RA, Hussey MG. Newborn phenylketonuria detection program in Massachusetts. Am J Pub Health 1964;54:2075-208I.

2. Guthrie R, Susi A.A simple phenyalanine method for detecting phenylketonuria in large populations of newborn infants. Pediatrics 1963;32:338-343.

3.Wilson JMG, Jungner F. Principles and practice of screening for disease. Public Health Papers. Geneva: Switzerland:World Health Organization, 1968:34.

4. Guthrie R. Screening "Inborn errors of metabolism" in the newborn infant-a multiple test program. JAMA 1968;4:92-98.

5. Sweetman L. Newborn screening by tandem mass spectrometry (MS/ MS). Clin Chem 1996;42:345-346.

6. Millington DS, Norwood DL, Kodo N.Application of fast atom bombardment with tandem mass spectrometry and liquid chromatography/mass spectrometry to the analysis of acylcarnitines in human urine, blood and tissue. Anal Biochem 1989;180:331-339. 7. Rashed MS, Ozand PT, Bucknall DN. Diagnosis of inborn errors of metabolism from blood spots by acylcarnitines and amino acids profiling using automated electrospray tandem mass spectrometry. Pediatr Res 1995;38:324-33|.

8. Hunt DF, Giordani ABB, Rhodes G, Herold DA. Mixture analysis by triple quadrupole mass spectrometry: metabolic profiling of urinary carboxylic acids. Clin Chem 1982;28:2387-2392.

9. Chace DH, Kalas TA.A biochemical perspective on the use of tandem mass spectrometry for newborn screening and clinical testing. Clin Biochem 2005;38:296-309.

10. Chace D. Use of tandem mass spectrometry for multianalyte screening of dried blood specimens from newborns. Clin Chem 2003;49(II):1797-|8I7.
II.Willey V, Carpenter K, Wilcken B. Newborn screening with tandem mass spectrometry: 12 months' experience in NSW Australia. Acta Paediatr 1999;(suppl 88):48-5I.

I2. Simonsen H, Jensen UG. Technical aspects of neonatal screening using tandem mass spectrometry. Report for 4th Meeting of the International Society for Neonatal Screening. Acta Paediatr 1999;432:52-54.

13. Velázquez A, Loera A,Aguirre BE, Gamboa S,Vargas H, Robles C. Tamiz neonatal para hipotiroidismo congénito y fenilcetonuria. Salud Publica Mex 1994;36:249-256.

I4. Ibarra I, Fernández C, Belmont L,Vela M. Elevada mortalidad y discapacidad en niños mexicanos con enfermedad de orina de jarabe de arce (EOJA). Gac Med Mex 2007;143(3): I97-20I.

15. Norma Oficial Mexicana NOM-034-SSA2-2002. Para la prevención y control de los defectos al nacimiento. Diario Oficial de la Federación, México DF, lunes 23 de octubre de 2003.

16. Millington D, Koeberl D. Metabolic screening in the newborn. GGH 2003;19(3):33-38.

17. Chace DH, Sherwin JE, Hillman S, Lorey F, Cunningham GC. Use of phenylalanine to tirosine ratio determined by tandem mass spectrometry to improve newborn screening for phenylketonuria of early discharge specimens collected in the first 24 hours. Clin Chem 1998;44:2405-2409. 18. Sweetman L. Newborn screening by tandem mass spectrometry: gaining experience. Clin Chem 2001;47:1937-1938.

19. Kaye Cl. Introduction to the newborn screening. Fact Sheets. Pediatrics 2006; | 18: 1304-13/2.

20. Rashed M. Clinical applications of tandem mass spectrometry: ten years of diagnosis and screening for inherited metabolic diseases. J Chromatogr B 2001;758:27-48.

21.Webster D, Dhondt JL, Hannon WH, Loeber G, Torresani T. Quality assurance and standardization: summary of the satellite meeting, Turku, Finland. Acta Paediatr 1999;(suppl 88):7-12.

22. Chace DH. Mass spectrometry in the clinical laboratory. Chem Rev 2001;101:445-477. 